\title{
Challenging the standard of care in advanced melanoma: focus on pembrolizumab
}

This article was published in the following Dove Press journal:

Cancer Management and Research

25 September 2017

Number of times this article has been viewed

\author{
Raghad M Abdul-Karim' \\ C Lance Cowey ${ }^{2-4}$ \\ 'Baylor Sammons Cancer Center, \\ ${ }^{2}$ Melanoma and Genitourinary \\ Oncology Program, Baylor University \\ Medical Center, Sammons Cancer \\ Center Texas Oncology, ${ }^{3}$ Baylor Skin \\ Malignancy Research and Treatment \\ Center, Baylor University Medical \\ Center, ${ }^{4}$ Developmental Therapeutics \\ Program, US Oncology Research, \\ Dallas, TX, USA
}

\begin{abstract}
The last several years have seen a dramatic rise in the number of effective therapies that have been shown to improve survival outcomes for patients with advanced melanoma. Among these treatments are the immune checkpoint inhibitors, a new class of immunotherapy, that have demonstrated the ability to improve both response rates and survival outcomes. Pembrolizumab, an immune checkpoint inhibitor that blocks the negative regulatory PD-1 receptor on T-cell lymphocytes, has shown improved efficacy compared to standard therapies with an acceptable tolerability profile. Additionally, this agent is being evaluated in adjuvant and combination trial strategies that have great potential to further advance outcomes. This review focuses on the advances that pembrolizumab has made in melanoma and what studies are upcoming that could change the future of melanoma treatment yet again.
\end{abstract}

Keywords: keytruda, MK-3475, immune checkpoint inhibitor, PD-1 inhibitor, adjuvant therapy, combination therapies, immunotherapy

\section{Introduction}

Malignant melanoma remains an important health concern with the incidence of melanoma rising faster than other malignancies with an estimated incidence of $>87,000$ new melanoma cases in the USA in $2017 .{ }^{1}$ It also remains a deadly disease with $\sim 10,000$ deaths annually associated with melanoma. Unlike the more common skin malignancies of squamous cell carcinoma and basal cell carcinoma, melanoma has far greater potential for distant spread, and when paired with poor sensitivity to conventional oncology treatments such as chemotherapy and radiation therapy, historically, clinical improvements in this disease have been quite challenging to see. For decades, only three therapies were US Food and Drug Administration (US FDA) approved for the disease, dacarbazine and interleukin-2 for unresectable metastatic disease and interferon for adjuvant treatment after surgery. Unfortunately, these drugs resulted in poor response rates $(\leq 10 \%)$ and no improvement in average survival..$^{2-4}$ The cytokines, interferon and interleukin-2, highlighted the potential for immunotherapy's role in treating melanoma; however, these early immunotherapy treatments had high toxicity rates, low response rates, and no improvement in the average survival of patients. ${ }^{2}$

Fortunately, since 2011, there have been advances in the treatment of advanced melanoma. ${ }^{5}$ These therapies have primarily come in two forms, genetically targeted therapies and immune modulating therapies. From the genomics standpoint, the $B R A F$ oncogene became a relevant treatment target after 2002 when it was found that $40-50 \%$ of melanoma tumors harbor BRAF V600 mutations. ${ }^{6}$ Several late phase
Correspondence: Charles Lance Cowey 3410 Worth Street, Suite 400, Dallas, TX 75426, USA

Tel + I 2/4370 I800

Email c.cowey@usoncology.com 
trials have shown significant improvement in response and survival outcomes with the use of BRAF and MEK inhibitors in patients with BRAF mutant metastatic melanoma. ${ }^{7-9}$ These new drugs include the BRAF inhibitors, vemurafenib and dabrafenib, as well as the MEK inhibitors, trametinib and cobimetinib. Recent studies have shown that blocking both the BRAF and MEK proteins in patients with BRAF mutant melanoma has resulted in superior outcomes compared to only BRAF blockade alone. ${ }^{10-12}$

In addition to genetically targeted therapies, immunotherapy has made a dramatic resurgence in the management of patients with advanced malignant melanoma. Previous work had shown that infiltration of tumor lymphocytes could be associated with improved outcomes; however, there were clear barriers to lymphocyte anticancer activities including lymphocyte regulation by tumors and other inflammatory cells. ${ }^{13}$ An important breakthrough was the identification and targeting of regulatory protein "switches" (called immune checkpoints) on the surface of T-cell lymphocytes. ${ }^{14}$ These immune checkpoints were found to have the ability to regulate lymphocyte function both in the early activation phase as well as inducing T-cell fatigue later during the immune process. The first immune checkpoint inhibitor that has been approved is ipilimumab, which is an antibody that targets the negative immune checkpoint cytotoxic lymphocyte antigen-4 (CTLA-4) on the T cells. ${ }^{15}$ Blocking CTLA-4 on the $\mathrm{T}$ cell promotes the activation and potentiation of these immune cells allowing them to have an immune cytotoxic effect on the cancer cells. Ipilimumab has been evaluated in two pivotal large Phase III trials, both of which have shown improvement in median overall survival (OS) in patients with metastatic melanoma, making it the first immunotherapy to ever do so. ${ }^{16,17}$ Although the improvement in average survival with ipilimumab was modest, there is a clear subset of patients, $\sim 18 \%$, who have prolonged survival benefit at the $8-10$ years timepoint. ${ }^{18}$ Ipilimumab has also been demonstrated to have potential for significant autoimmune toxicities in some patients, requiring careful monitoring and prompt intervention when side effects are detected. Common side effects have included fatigue, colitis, dermatitis, and immune-related endocrinopathies. Approximately 10-15\% of patients have experienced grade 3-4 immune-related toxicity in the standard $3 \mathrm{mg} / \mathrm{kg}$ dosing pivotal Phase III trial, and although most patients were able to achieve complete reversal of toxicity with steroid administration, a small percentage of patients required salvage infliximab for immune-related colitis and $1 \%$ of patients had immunerelated toxicity resulting in death.
Shortly, after ipilimumab was approved, the utility of another important immune checkpoint was successfully proven in clinical trials. The programmed death-1 (PD-1) checkpoint, which similar to CTLA-4 serves as a negative regulator of T-cell activity, has been shown to be quite relevant for drug development in melanoma. ${ }^{19-21}$ Mechanistically, when the PD-1 receptor binds to its ligand (programmed death-ligand 1 receptor [PD-L1]), an inhibitory signal is induced in $\mathrm{T}$ cells resulting in anergy and exhaustion. The ligand for PD-1 (PD-L1) is frequently expressed on the surface of melanoma cells and functions to help these cells escape immune surveillance. Two anti-PD-1 antibodies have been extensively studied and now FDA approved in melanoma, nivolumab and pembrolizumab. Blockade of PD-1 with monoclonal antibody allows activation and potentiation of T-cell lymphocytes, which can then result in clinically significant anticancer efficacy. Unique clinical features of both CTLA-4 and PD-1 inhibitor therapies have included the potential for delayed treatment responses (including early progression followed by response), pseudo-progression (tumor size increase due to inflammatory infiltrates), prolonged stable disease. and autoimmune side effects. ${ }^{22}$ Autoimmune side effects that have been observed with both CTLA-4 and PD-1 inhibitions are similar and include fatigue, colitis, dermatitis, hepatitis, and endocrinopathies among many other less common and rare inflammatory toxicities. However, an important distinguishing point between these two forms of immune checkpoint inhibitors is that side effects have tended to be of higher grade and frequency for ipilimumab compared to PD-1 inhibitors alone.

The development of BRAF-targeted therapies and immune checkpoint therapies has revolutionized the treatment landscape for melanoma. In particular, immune checkpoint inhibitors have demonstrated substantial potential for long-term survival in these patients fundamentally changing the field. The focus of this review is on the impact of the PD-1 antibody, pembrolizumab, which has been FDA approved for use in metastatic melanoma and is currently being studied in an array of clinical trials hoping to further advance clinical outcomes.

\section{PD-I pathway function}

PD-1 and other immune checkpoints play a fundamental role in immune response regulation. As shown in Figure 1, $\mathrm{T}$ lymphocytes are initially activated after the T-cell receptor (TCR) on the lymphocyte binds to antigen via the major histocompatibility complex (MHC) on antigen-presenting cells (APCs). In the case of T lymphocytes activated against 


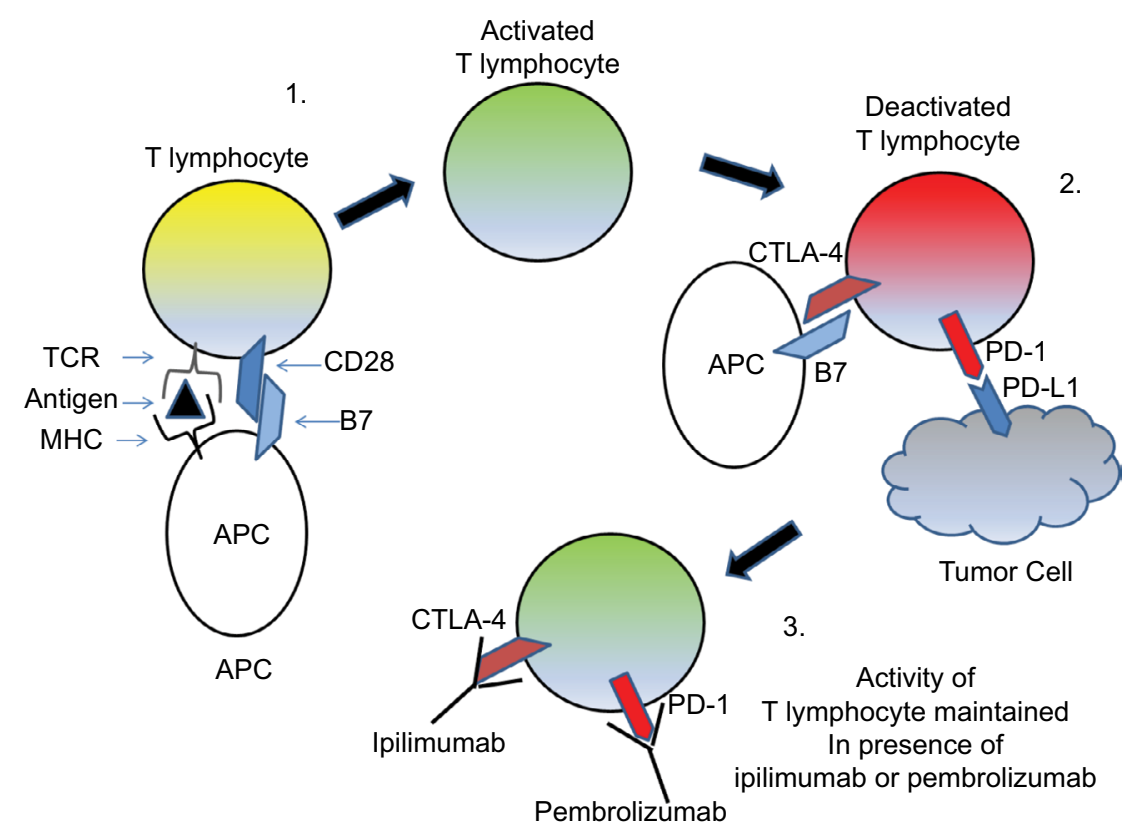

Figure I Diagram of T-lymphocyte activation and regulation by immune checkpoints.

Notes: I. The T lymphocyte is activated by the binding of the TCR to antigen presented by the MCH complex on APCs. This activation requires a costimulatory signal as depicted by the CD28:B7 binding. 2. The T cell can be inhibited by the expression of negative stimulatory receptors such as CTLA-4 and PD-I, also known as immune checkpoints. The binding of these immune checkpoints to their respective ligands results in deactivation of the T lymphocyte. 3. The monoclonal antibodies, ipilimumab and pembrolizumab, can maintain the activated lymphocyte by binding and impairing function of the immune checkpoints, CTLA-4 and PD-I, respectively. The active T lymphocyte is then able to potentiate the immune response and result in cancer cell killing.

Abbreviations: APCs, antigen-presenting cells; CTLA-4, cytotoxic lymphocyte antigen-4; MHC, major histocompatibility complex; PD-I, programmed death-I; PD-LI, programmed death-ligand I receptor; TCR, T-cell receptor.

melanoma cells, the antigen would be a mutated protein produced by the melanoma cell. The activation of the lymphocyte also requires a costimulatory signal such as $\mathrm{CD} 28$ (lymphocyte) and B7 (APC). From that point, the lymphocyte's activity is able to be further fine-tuned by signals from inhibitory and stimulatory immune checkpoint coreceptors expressed on the cell surface that regulate the magnitude and duration of its immune response. There are a variety of immune checkpoints with CTLA- 4 and PD-1 being negative regulators of lymphocyte function. ${ }^{23}$ The potential of the PD-1 receptors as an immune therapeutic target was supported by host of preclinical research. Initial work showed that aged PD-1 knockout mice spontaneously acquired autoimmune disease, providing proof of the receptor's role in immune response regulation. ${ }^{24}$ In addition, deficiency of PD-1 in mice enhances cytotoxic CD8 T-cell activity and increased their immune response to viral infection. ${ }^{23}$

The PD-1 receptor is expressed by activated T cells, natural killer cells, and myeloid cells and has two ligands: PD-L1 (or B7-H1) and PD-L2 (or B7-DC). The PD-1 ligand, PD-L1, varies in expression and location between different cells and is found to be highly expressed on tumor cells and virus-infected cells. Evaluation of PD-1 function in transgenically expressed PD-L1 tumor cells transplanted into mice has shown that, when PD-L1 is highly expressed within the tumor, there is an inhibition of CD8 T-cellmediated cytotoxicity and promotion of tumor growth..$^{25}$ This effect was reversed with the use of PD-1 antibody in this model. These findings support that tumors expressing PD-L1 have the potential to escape immune surveillance. Another means by which PD-1 activation can promote a protumor immune environment is the effect on CD28mediated costimulation. An imbalance of PD-L1 and the costimulatory B7.1 and B7.2 signals on APCs can affect the extent of T-cell activation and lead to a balance between tolerance and autoimmunity. ${ }^{25,26}$

The binding of PD-1 receptor to one of its two ligands, PD-L1 or PD-L2, results in a series of intracellular events culminating in the inhibition of TCR function and T-cell proliferation, thereby affecting the potential immune response. ${ }^{23}$ Additionally, activation of the PD-1 receptor by ligand binding affects lymphocyte function through a variety of intracellular pathways including inhibition of cell survival proteins (eg, BCL-2), decreased metabolic activity through downregulation of GLUT-1 transporter proteins, and altered cell cycle progression. ${ }^{27}$ These biological findings supported PD-1 and the PD-1 ligand as potential targets for immunotherapy drug development. 


\section{Early development of pembrolizumab}

Pembrolizumab (Keytruda ${ }^{\circledR}$, also previously known as MK-3475 and lambrolizumab; Merck \& Co., Inc., Kenilworth, NJ, USA) is a highly selective monoclonal IGg4-kappa isotype antibody that selectively binds to PD-1 blocking the receptor's negative impact on lymphocyte function. Of note, this IG4 subtype does not engage Fc receptors and, therefore, avoids the cytotoxic effects when it binds to PD-1 on the T cells. ${ }^{28}$ Pembrolizumab has been explored in a series of trials in patients with advanced melanoma (Table 1). It was first tested in a Phase I dose escalation study in patients with solid tumors, and it was deemed safe at dose levels of 1,3 , and $10 \mathrm{mg} / \mathrm{kg}$ (based on body weight) given every 2 weeks without exceeding the maximum tolerated dose. Positive clinical responses were observed at all dose levels used, particularly in those with melanoma. ${ }^{29}$

Critical to the evaluation and approval of pembrolizumab in melanoma was the Phase Ib Keynote-001 study. This trial investigated the safety and efficacy of different doses and schedules of pembrolizumab in a variety of cohorts in advanced melanoma patients..$^{30}$ The cohort that was pivotal to the initial FDA approval of pembrolizumab evaluated pretreated melanoma patients who had failed ipilimumab and BRAF-targeted therapy (if BRAF mutant). These patients were randomized to receive either pembrolizumab $2 \mathrm{mg} / \mathrm{kg}$ every 3 weeks ( $\mathrm{n}=89$ ) or $10 \mathrm{mg} / \mathrm{kg}$ every 3 weeks $(n=84)$. Patients were allowed to continue therapy until confirmed progressive disease (confirmed 4-6 weeks postinitial evidence of progression) or development of severe or intolerable toxicity. The primary efficacy outcomes for the trial were confirmed response rate and duration of response. Clinical characteristics of the patients in Keynote-001 demonstrated that most of the patients were heavily pretreated ( $73 \%$ with $\geq 2$ prior therapies) and had poor prognostic features such as M1c status (82\%). The confirmed overall response rate was similar between the groups at $24 \%$. Toxicity was deemed acceptable with common side effects including fatigue, nausea, cough, rash, and diarrhea. Grade $\geq 3$ side effects were uncommon, and $<10 \%$ of patients needed to stop therapy due to side effects. Based on this information, pembrolizumab was granted accelerated approval by the FDA in 2014.

Updated results of Keynote-001 including a pooled analysis of efficacy data in advanced melanoma patients have been published, demonstrating an overall response rate of $34 \%$, with the response being durable in $80 \%$ of these patients. ${ }^{31}$ Interestingly, the median OS for all patients treated with pembrolizumab in the trial was 23.8 months, and the 36 -month OS rate was $40 \%$ highlighting the long-term benefit of pembrolizumab. ${ }^{32}$ In comparison, advanced melanoma patients treated with ipilimumab had shown an average OS of 10-11 months in earlier Phase III studies ${ }^{33,34}$ and historic median survival prior to modern melanoma therapies has been in the range of 6-9 months. The findings from Keynote-001 supported the further exploration of pembrolizumab in patients with advanced melanoma.

Table I Pivotal trials of pembrolizumab supporting FDA approval in metastatic melanoma

\begin{tabular}{|c|c|c|c|c|c|c|}
\hline Clinical trial & $\mathbf{N}$ & Treatments & ORR & PFS & Median OS & $\begin{array}{l}\text { Grade } \geq 3 \\
\text { toxicity rate }\end{array}$ \\
\hline Keynote- $00 I^{30}$ & 173 & $\begin{array}{l}2 \mathrm{mg} / \mathrm{kg} \text { every } 3 \text { weeks } \\
(\mathrm{n}=89) \text { or } 10 \mathrm{mg} / \mathrm{kg} \\
\text { every } 3 \text { weeks }(\mathrm{n}=84)\end{array}$ & ORR: $34 \%$ & $35 \%$ 12-month PFS & $\begin{array}{l}\text { Median OS } \\
25.9 \text { months }\end{array}$ & $10 \%$ \\
\hline Keynote- $002^{33}$ & 540 & $\begin{array}{l}\text { Pembrolizumab } \\
10 \mathrm{mg} / \mathrm{kg} \text { every } 3 \text { weeks, } \\
\text { pembrolizumab } \\
2 \mathrm{mg} / \mathrm{kg} \text { every } 3 \text { weeks, } \\
\text { versus chemotherapy }\end{array}$ & $\begin{array}{l}\text { ORR: } 38,46 \text {, and } 8 \% \text {, } \\
\text { respectively } \\
\text { CR: } 2,3 \text {, and } 0 \% \text {, } \\
\text { respectively } \\
\text { PR: } 19,23 \text {, and } 4 \% \text {, } \\
\text { respectively }\end{array}$ & $\begin{array}{l}\text { 6-month PFS was } 34 \% \text { in } \\
\text { pembrolizumab } \\
2 \mathrm{mg} / \mathrm{kg} \text { group, } 38 \% \text { in the } \\
10 \mathrm{mg} / \mathrm{kg} \text { group, and } 16 \% \\
\text { in the chemotherapy } \\
\text { group }\end{array}$ & Not reached & $\begin{array}{l}\text { II- } 14 \% \text { in } \\
\text { pembrolizumab and } \\
26 \% \text { in chemotherapy }\end{array}$ \\
\hline Keynote- $006^{34}$ & 834 & $\begin{array}{l}10 \mathrm{mg} / \mathrm{kg} \text { every } 2 \text { weeks } \\
\text { versus every } 3 \text { weeks } \\
\text { versus ipilimumab }\end{array}$ & $\begin{array}{l}33.7 \text { and } 32.9 \% \text { for } \\
\text { pembrolizumab groups, } \\
\text { respectively, versus } \\
11.9 \% \text { for ipilimumab } \\
\text { CR rate of } 5-6 \% \text { in the } \\
\text { pembrolizumab arms and } \\
1.4 \% \text { in ipilimumab arm }\end{array}$ & $\begin{array}{l}\text { 5.5-month PFS for the } \\
\text { pembrolizumab every } \\
2 \text { weeks arm, } 4 \text {.I-month } \\
\text { PFS for pembrolizumab } \\
\text { every } 3 \text { weeks arm, and } \\
2.8 \text {-month PFS for the } \\
\text { ipilimumab arm }\end{array}$ & $\begin{array}{l}\text { I2-month OS rates } \\
\text { were } 74.1,68.4 \text {, and } \\
58.2 \% \text {, respectively } \\
24 \text {-month survival } \\
\text { rates of } 55.1,55.3 \text {, } \\
\text { and } 43.0 \% \text {, } \\
\text { respectively }\end{array}$ & $\begin{array}{l}10-13.3 \% \text { in the } \\
\text { pembrolizumab arms } \\
\text { and }-20 \% \text { in the } \\
\text { ipilimumab arm }\end{array}$ \\
\hline
\end{tabular}

Note: N, number of patients in study.

Abbreviations: CR, complete response; ORR, objective response rate; OS, overall survival; PFS, progression-free survival; FDA, Food and Drug Adminstration. 


\section{Pembrolizumab in comparative studies for advanced melanoma}

Following Keynote-001, two comparative studies in advanced melanoma were performed. The Keynote-002 trial explored the use of either pembrolizumab or investigator choice of cytotoxic chemotherapy in melanoma patients who were refractory to ipilimumab treatment. ${ }^{35}$ This Phase II trial enrolled 540 patients and randomized them equally to one of the three treatment arms, pembrolizumab $10 \mathrm{mg} / \mathrm{kg}$ every 3 weeks, pembrolizumab $2 \mathrm{mg} / \mathrm{kg}$ every 3 weeks, and chemotherapy (investigator chose between paclitaxel with carboplatin, paclitaxel, carboplatin, dacarbazine, or temozolomide). Stratification factors included lactate dehydrogenase (LDH) status, BRAF status, and Eastern Cooperative Oncology Group (ECOG) performance status. Although all patients were required to have prior ipilimumab, most were heavily pretreated with $>70 \%$ of patients receiving $\geq 2$ prior therapies (other treatments included interlukin-2 (IL-2), chemotherapy, and BRAF/MEK inhibitors). The primary endpoint of progression-free survival (PFS) was shown to be improved in the pembrolizumab arms compared to the chemotherapy arm. The 6-month PFS rate was $34 \%$ in the $2 \mathrm{mg} / \mathrm{kg}$ pembrolizumab arm, $38 \%$ in the $10 \mathrm{mg} / \mathrm{kg}$ pembrolizumab arm, and $16 \%$ in the chemotherapy arm. Post hoc analysis revealed a median PFS of 5.4, 5.8, and 3.6 months in the groups, respectively. Response rates among the arms were also improved in the pembrolizumab groups, with 21 and $25 \%$ of patients with RECIST-defined responses in the two pembrolizumab arms and $4 \%$ in the chemotherapy arm. Duration of response was not reached in the pembrolizumab arms at the time of publication. On Forest plot analysis of PFS across predefined subgroups, pembrolizumab was favored over chemotherapy in all categories, including age, sex, performance status, LDH status, and BRAF presence of mutation. Tolerability also was improved in the pembrolizumab arms as evidenced by fewer grade $\geq 3$ events, with a rate of $11-14 \%$ in the pembrolizumab arms and $26 \%$ in the chemotherapy arm. Discontinuation rates due to toxicity were 3 and $7 \%$ in the two pembrolizumab arms. Common side effects seen in the pembrolizumab groups included fatigue, pruritis, and rash. The results of the Keynote-002 trial further supported pembrolizumab as a standard of care option for advanced melanoma patients who have failed ipilimumab therapy.

Evaluation of pembrolizumab in ipilimumab-naive metastatic melanoma patients was done in the Keynote-006 study. ${ }^{36}$ In this Phase III trial, patients were randomized in a
$1: 1: 1$ ratio to receive pembrolizumab at a dose of $10 \mathrm{mg} / \mathrm{kg}$ every 2 weeks or every 3 weeks or ipilimumab at a dose of $3 \mathrm{mg} / \mathrm{kg}$ every 3 weeks for four cycles. Prior therapies were allowed in the study with the exclusion of prior PD-1, PD-L1, or CTLA-4 inhibitor therapies. Patients who had mutant BRAF were allowed to enroll without prior BRAF inhibitor therapy as long as LDH was not elevated. The intent of this criterion was to exclude those patients with potentially rapidly progressing disease that could be controlled with BRAFtargeted therapy. The trial had two coprimary endpoints of PFS and OS.

The study randomized 834 metastatic melanoma patients to therapy in the trial with key characteristics including two-thirds of patients being treatment naive, a third of patients having BRAF mutant melanoma, and two-thirds of patients with M1c status. Patients were required to have an ECOG status of 0 or 1 to enter the trial, with $70 \%$ entering having a performance status of 0 . PFS was found to be significantly prolonged in both of the pembrolizumab treatment arms compared to ipilimumab. The median PFS was 5.5 months for the pembrolizumab every 2 weeks arm, 4.1 months for the every 3 weeks arm, and 2.8 months for the ipilimumab arm. Additionally, landmark 6-month PFS rates were 47.3, 46.4, and 26.5\%, respectively. Objective response rates were also notably improved in the pembrolizumab arms at 33.7 and $32.9 \%$ compared to $11.9 \%$ for ipilimumab, respectively. Complete responses were seen in $5-6 \%$ of patients receiving pembrolizumab compared to $1.4 \%$ of patients receiving ipilimumab. The average time to response was similar in all groups (85-87 days). At the time of initial publication, median OS was not reached in any of the treatment arms; however, landmark 12-month OS rates were $74.1,68.4$, and $58.2 \%$, respectively. A follow-up presentation of OS data at ASCO 2016 reported 24-month survival rates of $55.1,55.3$, and $43 \%$, respectively. ${ }^{37}$

Toxicity also appeared improved in patients receiving pembrolizumab on the KEYNOTE-006 trial compared to ipilimumab-receiving patients. Grade $\geq 3$ adverse events were described as $10.1-13.3 \%$ in the pembrolizumab groups compared to $19.9 \%$ in the ipilimumab group. More patients required discontinuation due to toxicity from ipilimumab (9.4\%) compared to the pembrolizumab groups (4-6.9\%). Common toxicities from pembrolizumab included fatigue, diarrhea, rash, and pruritis. Based on the results of the KEYNOTE-006 study, pembrolizumab's approval was extended to the front-line setting for patients with metastatic melanoma, in addition to patients who had already progressed on prior ipilimumab or BRAF-targeted therapy. 


\section{Combination studies with pembrolizumab}

The CTLA-4 and PD-1 pathways appear to have complementary roles in suppressing T-cell function, both in the timing of their induction and the location of their activity. CTLA-4 has its function in the early stages of immune cell activation and primarily in the lymphatic tissues, whereas PD-1 function occurs later in the immune process resulting in lymphocyte exhaustion at the site of immune impact, for example, in the tumor microenvironment. Preclinical modeling showed higher levels of antitumor efficacy when both CTLA-4 and PD-1 pathways were inhibited. ${ }^{38}$ These findings led to a series of trials evaluating the role of ipilimumab and nivolumab in combination for advanced melanoma. A Phase I trial with this combination showed a response rate of $53 \%$ in the highest tolerable dose, with all patients having deep responses of $\geq 80 \%$ tumor burden size reduction. ${ }^{39}$ The combination treatment in this study also resulted in an increased immune toxicity profile with grade $\geq 3$ events in approximately half of patients. The results from this trial led to larger Phase II and Phase III randomized studies exploring this combination of checkpoint inhibitors. ${ }^{40,41}$ The Checkmate 067 Phase III trial explored the combination in untreated patients with stage III-IV unresectable melanoma. The study was designed as a double-blinded Phase III randomized trial where monotherapy with nivolumab or ipilimumab was compared with the combination of nivolumab and ipilimumab. The median PFS was 11.5 months for nivolumab and ipilimumab as compared to 2.9 months for ipilimumab alone and 6.9 months for nivolumab alone. Additionally, response rates were dramatically higher in the combination group (57.6\%) than in nivolumab (43.7\%) or ipilimumab (19\%). Immune-related toxicity was notable, with $36 \%$ of patients discontinuing therapy due to adverse events in the combination group. This study led to the FDA approval of this drug combination in 2015 based on encouraging results using these two check point inhibitors.

Subsequently, an interest in combining pembrolizumab with ipilimumab led to the Keynote-029 trial, which was designed to evaluate standard dose pembrolizumab with lower ipilimumab dosing in order to improve the toxicity profile. Data from an expansion cohort in the KEYNOTE-029 study have been recently presented. ${ }^{42}$ This cohort included 153 patients with advanced melanoma with no active brain metastasis and no prior immune checkpoint inhibitor therapy. The treatment scheme utilized was pembrolizumab $2 \mathrm{mg} /$ $\mathrm{kg}$ every 3 weeks and ipilimumab $1 \mathrm{mg} / \mathrm{kg}$ every 3 weeks for four doses followed by $2 \mathrm{mg} / \mathrm{kg}$ of pembrolizumab until intolerable toxicity, progression, or 2 years of therapy. Characteristics of these patients included $36 \%$ who carried the BRAF V600E mutation, $13 \%$ received $\geq 1$ prior therapy, and $12 \%$ received a prior BRAF \pm MEK inhibitor. Eightyfour percent of the patients had PD-L1-positive tumors (defined as $\geq 1 \%$ staining in tumor and adjacent immune cells). Adverse events related to immune activation of any grade were reported in $53 \%$ of the patients and $20 \%$ had a grade 3-4 severity. The overall response rate was $51 \%$, with $9 \%$ of patients achieving a complete remission. Given the encouraging efficacy and lower grade $3 / 4$ toxicity rate, further evaluation of this combination is expected.

In addition to the combination of available immune checkpoint inhibitors, many other strategies to explore the addition of novel agents to pembrolizumab are underway. Many of these combinations are in early phase testing; however, a few combinations have made it to late phases of clinical trial testing. Pembrolizumab is being studied in combination with talimogene laherperavec (also known as Imlygic or T-VEC) in the Masterkey-265 Phase Ib/III trial. T-VEC is an oncolytic virus, based on a modified herpes simplex strain, which has been shown to be effective in the management of advanced melanoma patients with superficial metastatic disease (eg, cutaneous and superficial nodal metastases). ${ }^{43}$ The virus is directly injected into melanoma tumors where it is able to infect and replicate in the cancerous cells resulting in cell lysis. The virus also has been designed to encode the granulocyte macrophage colony stimulating factor (GM-CSF) protein, which results in additional immune stimulus in the tumor microenvironment upon cell lysis. T-VEC has been approved for patients with metastatic melanoma and superficial injectable disease. Presentation of the Phase Ib portion of the Masterkey trial combining T-VEC with pembrolizumab was presented at ASCO in $2016 .{ }^{44}$ The combination had a confirmed response rate of $48 \%$ and a $33 \%$ grade $\geq 3$ toxicity rate. The trial has currently transitioned into a Phase III comparative study evaluating T-VEC with pembrolizumab versus pembrolizumab with placebo.

Another late phase study that has now completed enrollment and is awaiting results is the ECHO-301 trial (NCT02752074). This study is evaluating epacadostat, which is a novel immunomodulatory drug inhibiting activity of the indoleamine 2,3-dioxygenase (IDO) enzyme. IDO plays an important role in tryptophan metabolism in the tumor microenvironment and has been shown to support tumor immune escape. In a Phase I trial, epacadostat and pembrolizumab were studied in advanced solid tumors and included a melanoma cohort of patients. ${ }^{45}$ In the melanoma cohort ( $\left.n=19\right)$, the response rate was $57 \%$ and a disease control rate of $73 \%$ 
was observed. Common toxicities included fatigue, diarrhea, rash, arthralgias, and nausea, which are similar to pembrolizumab alone toxicities. The promising results of this study have led to the ECHO-301 trial, which has enrolled advanced melanoma patients to receive either pembrolizumab with epacadostat or pembrolizumab with placebo.

A variety of other trials evaluating pembrolizumab combination treatments are underway, including combinations with BRAF and MEK inhibitors, other unique immunecheckpoint inhibitors, vaccines, cytotoxic therapies, and radiation therapy (Table 2). Recent search on clinicaltrials. gov reveals $>70$ combination studies with pembrolizumab in melanoma alone. Based on pembrolizumab's efficacy and acceptable toxicity profile, it is easy to understand the rationale for wanting to combine it with other agents. However, identifying which are the most effective combinations and how to apply them clinically, based on individual patient tumor "immune phenotypes", will certainly be a challenging task for years to come.

\section{Pembrolizumab in the adjuvant setting}

Highly effective adjuvant therapy for high-risk resected stage II and III melanoma has been lacking for decades. In the 1990s, interferon was FDA approved based on improvement in recurrence-free survival (RFS) compared to placebo; however, no OS advantage has been seen with the drug. ${ }^{46}$ Subsequent studies of interferon evaluating various dosing schedules and combinations with other agents such as vaccines and cytotoxics also have failed to improve OS outcomes in randomized trials. Additionally, interferon has a high toxicity profile with poorly tolerated flu-like symptoms being common. A PEGylated form of interferon was FDA approved in 2011 based on improved RFS compared to placebo, but still there was no improvement survival; however, this new formulation is more convenient to administer. ${ }^{47}$

In 2015, results of the EORTC 18071 randomized trial of high-dose ipilimumab (10 mg/kg) versus placebo led to FDA approval with improvement in RFS noted, although at the cost of a high toxicity profile, with frequent grade 3/4 toxicities and a discontinuation rate of $\sim 50 \%{ }^{48} \mathrm{~A}$ subsequent update of OS showed ipilimumab demonstrating a 5-year absolute improvement in the survival of $11 \%$ compared to placebo and a $28 \%$ reduction in the risk of death hazard ratio ([HR] $0.78, P=0.001)$. Given the apparent superiority of the PD-1 inhibitors nivolumab and pembrolizumab over ipilimumab in trials of patients with metastatic melanoma, a series of new studies evaluating PD-1 inhibition in high-risk resected melanomas have been undertaken.

Table 2 Selected late phase trials of combination trials for advanced unresectable melanoma and Phase III adjuvant studies for resected high-risk melanoma

\begin{tabular}{|c|c|c|c|c|}
\hline Clinical trial & Study design & Study population & $\mathbf{N}$ & Primary endpoint \\
\hline $\begin{array}{l}\text { Keynote-022 } \\
\text { (NCT02 I 30466) }\end{array}$ & $\begin{array}{l}\text { Phase I/II trial multiple cohort study evaluating } \\
\text { dabrafenib/trametinib/pembrolizumab } \\
\text { combination therapy in patients with advanced } \\
\text { BRAF mutant melanoma and trametinib/ } \\
\text { pembrolizumab in patients with advanced } \\
\text { non-BRAF mutant melanoma }\end{array}$ & $\begin{array}{l}\text { Metastatic unresectable melanoma; } \\
\text { immune checkpoint inhibitor and } \\
\text { BRAF/MEK inhibitor naive }\end{array}$ & 219 & $\begin{array}{l}\text { DLT frequency in } \\
\text { Phase I portion; PFS in } \\
\text { Phase II portion }\end{array}$ \\
\hline $\begin{array}{l}\text { Keynote-034 } \\
\text { (NCT02263508) }\end{array}$ & $\begin{array}{l}\text { Phase Ib/III trial of T-VEC + pembrolizumab } \\
\text { versus placebo + pembrolizumab }\end{array}$ & $\begin{array}{l}\text { Metastatic unresectable melanoma; } \\
\text { no prior immune checkpoint } \\
\text { therapy; BRAF mutant melanoma } \\
\text { patients are allowed to have had } \\
\text { BRAF or MEK inhibitor therapy }\end{array}$ & 660 & $\begin{array}{l}\text { DLT frequency (Phase } \\
\text { lb portion); PFS and } \\
\text { OS (Phase III portion) }\end{array}$ \\
\hline $\begin{array}{l}\text { Keynote-252/ } \\
\text { ECHO-30I } \\
\text { (NCT02752074) }\end{array}$ & $\begin{array}{l}\text { Phase III study of pembrolizumab plus } \\
\text { epacadostat versus pembrolizumab plus } \\
\text { placebo in unresectable or metastatic melanoma }\end{array}$ & $\begin{array}{l}\text { Metastatic unresectable melanoma; } \\
\text { treatment naive }\end{array}$ & 600 & PFS and OS \\
\hline $\begin{array}{l}\text { Keynote-054 } \\
\text { (NCT02362594) }\end{array}$ & $\begin{array}{l}\text { Phase III study of adjuvant pembrolizumab } \\
\text { versus placebo in resected, high-risk melanoma }\end{array}$ & $\begin{array}{l}\text { Completely resected stage IIIA/B/C } \\
\text { (>I mm node involvement); } \\
\text { adjuvant treatment setting }\end{array}$ & 900 & $\begin{array}{l}\text { RFS; RFS in PD-LI- } \\
\text { expressing patients }\end{array}$ \\
\hline SI 404 (NCT02506I53) & $\begin{array}{l}\text { Phase III study of adjuvant pembrolizumab } \\
\text { versus high-dose interferon or ipilimumab in } \\
\text { resected, high-risk melanoma }\end{array}$ & $\begin{array}{l}\text { Completely resected stage IIIA/B/C, } \\
\mathrm{IV} \text {; adjuvant treatment setting }\end{array}$ & $\mathrm{I}, 378$ & $\begin{array}{l}\text { OS; RFS; OS based } \\
\text { on PD-LI expression; } \\
\text { RFS based on PD-LI } \\
\text { expression }\end{array}$ \\
\hline
\end{tabular}

Note: N, number of patients to be enrolled in study.

Abbreviations:, DLT, dose-limiting toxicity; PFS, progression-free survival; OS, overall survival; RFS, recurrence-free survival; PD-LI, programmed death-ligand I receptor; T-VEC, talimogene laherperavec. 
Pembrolizumab is being evaluated in two separate randomized Phase III trials. In Keynote-054, pembrolizumab is being compared to placebo in patients with IIIA ( $>1 \mathrm{~mm}$ involvement in lymph node), IIIB, and IIIC melanoma, which has been completely resected. RFS in all the subjects and in the subset of subjects with PD-1 ligand expression in the resected tumor is the primary endpoint. Secondary endpoints include distant metastatic-free survival and OS. This study has completed accrual and is awaiting data maturation for publication. In a separate adjuvant study conducted in the cooperative group setting, S1404 is evaluating pembrolizumab compared to investigator's choice of high-dose interferon or high-dose ipilimumab for resected high-risk stage III melanoma (stages IIIA, IIIB, IIIC, and IV). Baseline PD-1 ligand testing of the tumor is being performed, and patients are being stratified by expression status. Primary endpoints include OS, RFS, and PD-L1 expression status. ${ }^{49}$ Of note, similar studies with nivolumab are also being conducted but not yet reported. Hopefully, these trials with PD-1 inhibitors will further advance outcomes for patients with high-risk melanoma who currently have limited options in this setting.

\section{Biomarkers for pembrolizumab in melanoma}

With the advent of effective therapy for melanoma, the development and validation of predictive biomarkers has been an important goal of the last several years. The discovery of a single or group of markers predicting response could help define a subset of patients who are more likely to benefit from a particular immunotherapy or combination of therapies. A validated biomarker could also help save a person from exposure to unnecessary cost and toxicity if a treatment is known to unlikely be beneficial. For single agent PD-1 inhibition, such as pembrolizumab therapy, the expression of PD-ligand expression in the tumor was a clear initial candidate for biomarker development. The hypothesis would be that if a patient's melanoma tumor expresses ligand, which activates the PD- 1 pathway, then perhaps this is a relevant therapeutic target for their disease. Tumor cell and intratumoral immune cell immunohistochemical expression of PD-1 ligand has been evaluated as a biomarker in the pivotal studies with pembrolizumab and has been a stratification factor in randomized trials. In the Phase III comparison study of pembrolizumab and ipilimumab, PD-1 ligand tumor expression was evaluated with $\sim 80 \%$ of patients in the study defined as positively expressing the ligand $(\geq 1 \%$ staining cutoff for positive). In this study, there was a PFS benefit of pembrolizumab over ipilimumab regardless of PD-1 ligand expression pattern. Albeit it was a small subset, evaluation of OS in the PD-1 ligand-negative group showed no difference in OS in subgroup analysis between the arms, suggesting that these patients do just as well with PD-1 inhibition or ipilimumab. There have been a variety of pitfalls to PD-1 ligand expression as an effective biomarker including a disputable expression percentage cutoff, whether to measure tumor cell or immune cell expression, heterogeneity of expression both intratumorally as well as among different metastatic tumors, and a variety of different antibodies used for expression analysis. Finally, there are a significant percentage of patients with PD-1 ligand nonexpressing tumors, no matter the cutoff implemented, who benefit from PD-1 inhibitor-based therapy.

Given the basic biology of immune function with the recognition of antigen as an important trigger for activation of a sustainable immune response, evaluation of tumor antigen production has been another promising biomarker candidate. Melanomas are known to have a very high mutational load, with ultraviolet (UV) radiation inducing hundreds to thousands of point mutations in tumor cell DNA. ${ }^{50}$ In an exploratory study of patients treated with ipilimumab, whole genome sequencing was done on melanoma tumor samples and matched blood samples to analyze somatic mutations and the neoantigens generated from these mutations. Researchers found that the mutational load and specific neoantigen expression by tumor cells were linked to a benefit from CTLA-4 blockade in these patients with melanoma. ${ }^{51}$ This concept of higher mutational load creating a broad neoantigen landscape was found in patients with non-small-cell lung cancer as well. ${ }^{52}$ Mutational load and neoantigen profiling have become the most intriguing biomarker candidates, which have made their way into several prospective melanoma studies.

An additional candidate biomarker profile has been to evaluate the patterns of immune cell infiltration into melanoma tumors. Taube et $\mathrm{al}^{53}$ found that infiltrating immune cells were geographically associated with PD-L1 expression and that immune activity in the tumor milieu was associated with PD-L1 expression in tumor cells and tumor immune infiltrates. Importantly, tumor cell PD-L1 expression correlated highly with the response to anti-PD-1 treatment. ${ }^{53}$ Another report reported the correlation between tumor infiltrating CD8+ $\mathrm{T}$ cells (killer lymphocytes) and tumor radiographic response. ${ }^{54}$ Patients with higher pretreatment CD8 cell infiltration, as well as PD-1 and PD-L1 expression both inside the tumor and at the tumor margins, had a higher radiographic response. ${ }^{54}$ In the same report, the pretreatment density of CD8+ T cells was closely associated with clinical response to $\mathrm{PD}-1$ blockade. 
Ongoing comparative and combination trials are evaluating many of these new potential biomarker candidates either in all or in a subset of the trial population. This is critically important to build datasets that can tease out the most relevant biomarker to help select appropriate therapy in the future.

\section{Conclusion}

Pembrolizumab has become a critical addition to the therapeutic options for the management of melanoma. With a series of clinical trials, which have supported its role as an effective and tolerable agent compared to other standard therapies, it has gained FDA approval and widespread use in patients with advanced or metastatic melanoma. Pembrolizumab has been shown to improve survival outcomes for patients with advanced melanoma making it one of just a handful of agents to be able to do so in this disease. Currently, it is approved in the USA for front-line use in metastatic melanoma and in patients previously treated with ipilimumab and/or BRAF-targeted therapy.

Despite the effectiveness of this new generation of agents, a large subset of patients will still succumb to this disease, which demands further research to improve patient selection for best therapy and evaluation of more broadly effective combinations. The specific utilization of pembrolizumab in melanoma therapy may change in the future to meet these goals as several early combination trials with the agent have shown great potential to be more effective and not sacrifice tolerability. In fact, multiple combinations with a variety of agents could result in positive trial findings, making biomarker discovery and validation critical to identifying what patient population may benefit the most from a particular combination therapy. Despite the large task ahead to further improve therapeutic outcomes, the rapid advances in just the last few years for this disease including the development of pembrolizumab give great hope that these goals are achievable in the near future.

\section{Disclosure}

CLC received clinical research funding from Merck, BMS, Novartis, Genentech, and Amgen. The authors report no other conflicts of interest in this work.

\section{References}

1. American Cancer Society. Cancer Facts \& Figures 2017. Atlanta, GA: American Cancer Society; 2017.

2. Atkins MB, Kunkel L, Sznol M, Rosenberg SA. High-dose recombinant interleukin-2 therapy in patients with metastatic melanoma: long-term survival update. Cancer J Sci Am. 2000;6(suppl 1):S11-S14.

3. Anderson CM, Buzaid AC, Legha SS. Systemic treatments for advanced cutaneous melanoma. Oncology (Williston Park). 1995;9:1149-1158. [discussion 1163-1164, 1167-1168].
4. Atkins MB, Lotze MT, Dutcher JP, et al. High-dose recombinant interleukin 2 therapy for patients with metastatic melanoma: analysis of 270 patients treated between 1985 and 1993. J Clin Oncol. 1999;17(7): 2105-2116.

5. John L, Cowey CL. The rapid emergence of novel therapeutics in advanced malignant melanoma. Dermatol Ther (Heidelb). 2015;5(3): 151-169.

6. Davies H, Bignell GR, Cox C, et al. Mutations of the BRAF gene in human cancer. Nature. 2002;417(6892):949-954.

7. Hauschild A, Grob JJ, Demidov LV, et al. Dabrafenib in BRAF-mutated metastatic melanoma: a multicentre, open-label, phase 3 randomised controlled trial. Lancet. 2012;380(9839):358-365.

8. Chapman PB, Hauschild A, Robert C, et al; BRIM-3 Study Group. Improved survival with vemurafenib in melanoma with BRAF V600E mutation. N Engl J Med. 2011;364(26):2507-2516.

9. Flaherty KT, Robert C, Hersey P, et al; METRIC Study Group. Improved survival with MEK inhibition in BRAF-mutated melanoma. $N$ Engl J Med. 2012;367(2):107-114.

10. Flaherty KT, Infante JR, Daud A, et al. Combined BRAF and MEK inhibition in melanoma with BRAF V600 mutations. $N$ Engl J Med. 2012;367(18):1694-1703.

11. Ascierto PA, McArthur GA, Dreno B, et al. coBRIM: a phase 3, doubleblind, placebo-controlled study of vemurafenib versus vemurafenib + cobimetinib in previously untreated BRAF (V600) mutation-positive patients with unresectable locally advanced or metastatic melanoma (NCT01689519). J Transl Med. 2015;13:2061.

12. Robert C, Karaszewska B, Schachter J, et al. Improved overall survival in melanoma with combined dabrafenib and trametinib. N Engl J Med. 2015;372(1):30-39.

13. Pastorfide GC, Kibbi AG, de Roa AL, et al. Image analysis of stage 1 melanoma (1.00-2.50 mm): lymphocytic infiltrates related to metastasis and survival. J Cutan Pathol. 1992;19(5):390-397.

14. Pardoll DM. The blockade of immune checkpoints in cancer immunotherapy. Nat Rev Cancer. 2012;12(4):252-264.

15. Fong L, Small EJ. Anti-cytotoxic T-lymphocyte antigen-4 antibody: the first in an emerging class of immunomodulatory antibodies for cancer treatment. J Clin Oncol. 2008;26(32):5275-5283.

16. Hodi FS, O'Day SJ, McDermott DF, et al. Improved survival with ipilimumab in patients with metastatic melanoma. $N$ Engl J Med. 2010;363(8):711-723.

17. Robert C, Thomas L, Bondarenko I, et al. Ipilimumab plus dacarbazine for previously untreated metastatic melanoma. $N$ Engl J Med. 2011;364(26):2517-2526.

18. Schadendorf D, Hodi FS, Robert C, et al. Pooled analysis of long-term survival data from phase II and phase III trials of ipilimumab in unresectable or metastatic melanoma. J Clin Oncol. 2015;33(17):1889-1894.

19. Brahmer JR, Drake CG, Wollner I, et al. Phase I study of single-agent anti-programmed death-1 (MDX-1106) in refractory solid tumors: safety, clinical activity, pharmacodynamics, and immunologic correlates. J Clin Oncol. 2010;28(19):3167-3175.

20. Brahmer JR, Tykodi SS, Chow LQ, et al. Safety and activity of anti-PD-L1 antibody in patients with advanced cancer. N Engl J Med. 2012;366(26): 2455-2465.

21. Topalian SL, Hodi FS, Brahmer JR, et al. Safety, activity, and immune correlates of anti-PD-1 antibody in cancer. NEngl J Med. 2012;366(26): 2443-2454.

22. Wolchok JD, Hoos A, O'Day S, et al. Guidelines for the evaluation of immune therapy activity in solid tumors: immune-related response criteria. Clin Cancer Res. 2009;15(23):7412-7420.

23. Okazaki T, Chikuma S, Iwai Y, Fagarasan S, Honjo T. A rheostat for immune responses: the unique properties of PD-1 and their advantages for clinical application. Nat Immunol. 2013;14(12):1212-1218.

24. Nishimura H, Nose M, Hiai H, Minato N, Honjo T. Development of lupus-like autoimmune diseases by disruption of the PD-1 gene encoding an ITIM motif-carrying immunoreceptor. Immunity. 1999;11(2):141-151. 
25. Iwai Y, Ishida M, Tanaka Y, Okazaki T, Honjo T, Minato N. Involvement of PD-L1 on tumor cells in the escape from host immune system and tumor immunotherapy by PD-L1 blockade. Proc Natl Acad Sci U S A. 2002;99(19):12293-12297.

26. Freeman GJ, Long AJ, Iwai Y, et al. Engagement of the PD-1 immunoinhibitory receptor by a novel $\mathrm{B} 7$ family member leads to negative regulation of lymphocyte activation. J Exp Med. 2000;192(7):1027-1034.

27. Dong Y, Sun Q, Zhang X. PD-1 and its ligands are important immune checkpoints in cancer. Oncotarget. 2017;8(2):2171-2186.

28. Hamid O, Robert C, DaudA, et al. Safety and tumor responses with lambrolizumab (anti-PD-1) in melanoma. N Engl J Med. 2013;369(2):134-144.

29. Patnaik A, Kang SP, Rasco D, et al. Phase I study of pembrolizumab (MK-3475; Anti-PD-1 monoclonal antibody) in patients with advanced solid tumors. Clin Cancer Res. 2015;21:4286-4293.

30. Robert C, Ribas A, Wolchok JD, et al. Anti-programmed-death-receptor-1 treatment with pembrolizumab in ipilimumab-refractory advanced melanoma: a randomised dose-comparison cohort of a phase 1 trial. Lancet. 2014;384(9948):1109-1117.

31. Ribas A, Hodi S, Kefford R, et al. Efficacy and safety of the anti-PD-1 monoclonal antibody MK-3475 in 411 patients (pts) with melanoma (MEL). J Clin Oncol. 2014;32(suppl):abstrLBA9000.

32. Robert C, Ribas A, Hamid O. Three-year overall survival for patients with advanced melanoma treated with pembrolizumab in KEYNOTE-001. J Clin Oncol. 2016;34:abstract9503.

33. Robert $\mathrm{C}$, Thomas L, Bondarenko I et al. Ipilimumab plus dacarbazine for previously untreated melanoma. New England Journal of Medicine. 2011;364(26);2517-26.

34. Hodi S, O’Day SJ, McDermott, DF et al. Improved survival with ipilimumab in patients with metastatic melanoma. New England Journal of Medicine. 2010;363(8);711-23.

35. Ribas A, Puzanov I, Dummer R, et al. Pembrolizumab versus investigator-choice chemotherapy for ipilimumab-refractory melanoma (KEYNOTE-002): a randomised, controlled, phase 2 trial. Lancet Oncol. 2015;16(8):908-918.

36. Robert C, Schachter J, Long GV, et al; KEYNOTE-006 Investigators. Pembrolizumab versus ipilimumab in advanced melanoma. $N$ Engl J Med. 2015;372(26):2521-2532.

37. Schachter J, Ribas A, Long A. Pembrolizumab versus ipilimumab for advanced melanoma: final overall survival analysis of KEYNOTE-006. J Clin Oncol. 2016;34:9504.

38. Curran MA, Montalvo W, Yagita H, Allison JP. PD-1 and CTLA-4 combination blockade expands infiltrating $\mathrm{T}$ cells and reduces regulatory $\mathrm{T}$ and myeloid cells within B16 melanoma tumors. Proc Natl Acad Sci U S A. 2010;107(9):4275-4280.

39. Wolchok JD, Kluger H, Callahan MK, et al. Nivolumab plus ipilimumab in advanced melanoma. N Engl J Med. 2013;369(2):122-133.

40. Larkin J, Chiarion-Sileni V, Gonzalez R, et al. Combined nivolumab and ipilimumab or monotherapy in untreated melanoma. NEngl JMed. 2015;373(1):23-34.
41. Postow MA, Chesney J, Pavlick AC, et al. Nivolumab and ipilimumab versus ipilimumab in untreated melanoma. $N$ Engl J Med. 2015;372:2006-2017.

42. Long GV, Atkinson V, Cebon JS, et al. Pembrolizumab (pembro) plus ipilimumab (ipi) for advanced melanoma: results of the KEYNOTE-029 expansion cohort. J Clin Oncol. 2016;34(suppl):abstr9506.

43. Andtbacka RH, Kaufman HL, Collichio F, et al. Talimogene laherparepvec improves durable response rate in patients with advanced melanoma. J Clin Oncol. 2015;33(25):2780-2788.

44. Long G, Dummer R, Ribas A. Efficacy analysis of MASTERKEY-265 phase $1 \mathrm{~b}$ study of talimogene laherparepvec (T-VEC) and pembrolizumab (pembro) for unresectable stage IIIB-IV melanoma. J Clin Oncol. 2016;34:abstract9568.

45. Gandadhar T, Hamid O, Smith DC. Epacadostat plus pembrolizumab in patients with advanced melanoma and select solid tumors: updated phase 1 results from ECHO-202/KEYNOTE-037. Ann Oncol. 2016;27: 1110D.

46. Kirkwood JM, Strawderman MH, Ernstoff MS, Smith TJ, Borden EC, Blum RH. Interferon alfa-2b adjuvant therapy of high-risk resected cutaneous melanoma: the Eastern Cooperative Oncology Group Trial EST 1684. J Clin Oncol. 1996;14(1):7-17.

47. Eggermont AM, Suciu S, Testori A, et al. Long-term results of the randomized phase III trial EORTC 18991 of adjuvant therapy with pegylated interferon alfa-2b versus observation in resected stage III melanoma. J Clin Oncol. 2012;30(31):3810-3818.

48. Eggermont AM, Chiarion-Sileni V, Grob JJ, et al. Adjuvant ipilimumab versus placebo after complete resection of high-risk stage III melanoma (EORTC 18071): a randomised, double-blind, phase 3 trial. Lancet Oncol. 2015;16(5):522-530.

49. Grossmann KF, Othus M, Tarhini AA, et al. SWOG S1404: a phase III randomized trial comparing standard of care adjuvant therapy to pembrolizumab in patients with high risk resected melanoma. J Clin Oncol. 2016;34(suppl):e21032.

50. Davar D, Lin Y, Kirkwood JM. Unfolding the mutational landscape of human melanoma. J Invest Dermatol. 2015;135(3):659-662.

51. Snyder A, Makarov V, Merghoub T, et al. Genetic basis for clinical response to CTLA-4 blockade in melanoma. NEngl J Med. 2014;371(23): 2189-2199.

52. Rizvi NA, Hellmann MD, Snyder A, et al. Cancer immunology. Mutational landscape determines sensitivity to PD-1 blockade in non-small cell lung cancer. Science. 2015;348(6230):124-128.

53. Taube JM, Klein A, Brahmer JR, et al. Association of PD-1, PD-1 ligands, and other features of the tumor immune microenvironment with response to anti-PD-1 therapy. Clin Cancer Res. 2014;20(19):5064-5074.

54. Tumeh PC, Harview CL, Yearley JH, et al. PD-1 blockade induces responses by inhibiting adaptive immune resistance. Nature. 2014;515(7528): 568-571.
Cancer Management and Research

\section{Publish your work in this journal}

Cancer Management and Research is an international, peer-reviewed open access journal focusing on cancer research and the optimal use of preventative and integrated treatment interventions to achieve improved outcomes, enhanced survival and quality of life for the cancer patient. The manuscript management system is completely online and includes

\section{Dovepress}

a very quick and fair peer-review system, which is all easy to use. Visit http://www.dovepress.com/testimonials.php to read real quotes from published authors. 